\title{
A Path Integral Generalization of Bell Local Hidden Variable Models for Unstable Particles
}

\author{
Gianpaolo Bei \\ I.I.S.S. Charles Darwin, Rome, Italy \\ Email: capo.gian@libero.it
}

How to cite this paper: Bei, G. (2021) A Path Integral Generalization of Bell Local Hidden Variable Models for Unstable Particles. Journal of Applied Mathematics and Physics, 9, 2430-2438. https://doi.org/10.4236/jamp.2021.910154

Received: September 6, 2021

Accepted: October 9, 2021

Published: October 12, 2021

Copyright $\odot 2021$ by author(s) and Scientific Research Publishing Inc. This work is licensed under the Creative Commons Attribution International License (CC BY 4.0).

http://creativecommons.org/licenses/by/4.0/

\section{(c) (i) Open Access}

\begin{abstract}
We discuss the problem of the generalization of Bell local hidden variable models for unstable particles as nucleons or decaying quantum bound states. We propose to extend the formalism of real deterministic hidden variables in the complex domain, in analogy with the quantum Gamow ket formalism, and we introduce a time dependent classical probability density distribution by which we implement hidden time dependence in the quantum expectation values. We suggest therefore a classical framework which may recover by asymptotic temporal limits the standard Bell stationary quantum statistical averages. Endly we discuss the possible relevance of our proposal for general non-isolated quantum systems in noninertial frames and the consequent dynamic effects of vacuum instabilities on E.P.R. tests and Q.M. ensemble statistical averages.
\end{abstract}

\section{Keywords}

Entangled Unstable Particles, Complex Hidden Variables, Path Dependent Expectation Values, Time Dependent Bell Inequalities

\section{Introduction}

Since the pioneer work of Einstein, Podolsky and Rosen [1] against the standard Copenhagen interpretation of Quantum Mechanics on hidden variables have been debating the presumed incompleteness of quantum microscopic systems studying the dynamics of stable free particles like electrons. This first proposal was not aimed to show the possibility to describe by a classical model the reality of the trajectory of a bound electron or that one of a decaying nucleus but was intended to show how to measure simultaneously the position and momentum of a beam of entangled electrons [2]. Despite it was an alternative proposal to bypass Heisenberg uncertainty principle it was continued to be used the stan- 
dard wavefunction collapse postulate (supposed to be instantaneous) and the Born probability interpretation of the quantum wavefunctions [3].

This model showed that the assumption that Quantum Mechanics describes completely quantum states implies the absurd consequence of "spooky action at distance" between entangled and well separated electrons and therefore they suggested to abandon the epistemic statistical interpretation of Quantum Mechanics. This work, even if it didn't context specific experimental predictions of the standard formalism, inspired the hidden variable model of Bohm [4].

This author simplified the original E.P.R. model, introducing the seminal idea to substitute the momentum entanglement with the spin entanglement of electron pairs, paving the way to the extension of hidden variable models for photon spin polarization pairs (which has been till now the most experimentally tested formulation of hidden variable models). Bohm, even if convinced of the importance of the Einstein belief on the necessity to give a space-time description of electron dynamics and electron spin (notwithstanding the Pauli strong opposition against this realistic point of view), focused his original proposal on the problem of the expectation values of quantum singlet states, described by its hidden variables (assuming the Bohr hypothesis of uncontrollable effect of the apparatus on the system). This quantum singlet state framework was then adapted from Bell for deducing his famous theorem [5].

Curiously during the thirty years, that started with the EPR paper to the celebrated discovery of Bell inequalities, particle physics discovered a lot of unstable particles and was developed a theory of nuclear fission [6] (based on classical concepts of nuclei traiectories) which didn't attract the attention of Einstein and his followers. Notwithstanding the formalism developed by Gamow for nuclear physics [7] described these particles states with wavefunctions which didn't belong to Hillbert space (and that were not eigenstates of hermitian operators) it was applied to them the Born probability interpretation and the wave function collapse postulate to make predictions that were then successfully experimentally tested.

Strangely this contradictory approach, based on Gamow kets on the one hand and on the Schrodinger equation dynamics and Pauli spin operators on the other, was not strongly outlined by the famous opponents of the Copenhagen interpretation and it was not considered as a new theoretical and experimental context suitable to develop classical deterministic models of quantum accelerated systems as unstable nucleons or excited quasi bound states of atoms and ions.

On our opinion, it is possible to implement this research program for every non-isolated quantum system exploiting some ideas developed in three previous papers of the author [8] [9] [10] introducing time dependent complex hidden variables (either scalars, vectors or matrices as moments of inertia) and generalizing the standard integral on the probability density distribution as a complex path dependent one.

This novel tentative approach that we propose to generalize hidden variable 
theories, which will be explained more deeply in the next paragraph, shares with some recent papers [11] [12] [13] the view that Bell's Theorem successful experimental tests are a by-product of the non-sinusoidal behaviour of all quantum two-particle spin correlations. Differently from these authors we don't consider projected spin of unstable particles as dichotomic real variables but as complex time dependent vectors which makes the Bell correlation functions and Bell inequalities dependent on time dependent complex angles; we propose, more generally, to explain every E.P.R. experiment for entangled open quantum systems with this non-sinusoidal two particle correlation functions. Therefore our alternative framework conceives each quantum particle, either free or bound state, as an unstable state with hidden time dependent energy bandwidth and complex variable magnetic moment (and variable imaginary mass) caused by its intrinsic coupling with the fields of surrounding particles and the induced vacuum fluctuations. We wish to remark that, as some authors recently argued [14] [15], the original aim of the realistic view of Einstein, strongly contrasted by the epistemic probabilistic approach of Bohr and Heisenberg uncertainty relations for non-commuting operators, was to give a real static numerical value to each observable of a quantum system prior to its interaction with the apparatus (Einstein idea of hidden variable is, we think, an analogue of a hidden initial conditions for Newtonian classical differential equation); this assumption, however, doesn't imply that these values have to be real ones, as, on the contrary, was implicitly implemented in the deterministic model of Bohm [4], and subsequently was used from Bell to deduce his inequalities, assuming that its restricted family of local hidden variable models had rotational symmetry and dicotomic projected spin values on the singlet state( without this hypothesis the Bell correlation functions are no more cosinusoidal [5]. In the following paragraph, we will expose our alternative proposal and in the last paragraph, we will discuss its possible extension to general open quantum systems and suggest some possible experiments to test it.

\section{Model}

Our proposal will not consider entanglement polarizations of photon pairs since we think that they concern Q.E.D. quantum states and the controversial hypothesis of photon indivisibility; in fact we think that photon entanglement should be based on generalization of the Bell inequalities to entanglement of Fock states (which needs an infinite dimensional path integral generalizations of classical ensemble averages); over more to define perfect two-photon entangled polarized state, it is necessary to assume stability of the photon vacuum state, considering irrelevant the effect of background fields and inertial forces of the rotating Earth.

We prefere instead to study the standard formalism of singlet state of two spin entangled systems which can be exploited to describe bound states of unstable nucleons or bound states of atoms or molecules [16]. 


$$
\Psi=\frac{1}{\sqrt{2}}\left[\Psi_{+}(1) \Psi_{-}(2)-\Psi_{-}(1) \Psi_{+}(2)\right]
$$

where the factorized wavefunctions $\Psi$, belonging to a separable Hilbert space, are usually relative to one electron state with spin up or down along a predefine direction with respect to the chosen magnetic axis of a Stern-Gerlach apparatus. We want to note that this framework is based on the hidden assumption that this singlet quantum system is suitable for describing isolated entangled particles, assuming that the zero intrinsic total angular momentum is conserved in the future if its dynamics are supposed to have rotational symmetry (that is equivalent to exclude any hidden spin-orbit coupling neither with the environment nor with the apparatus). We think that this approach is not realistic when it is applied to accelerated and deformable nucleons produced by the decay of unstable nuclei (making the spin quantized dichotomic variable not well justified for time dependent magnetic dipole of accelerated nucleons or ions); in particular we believe it is necessary to make time dependent the expectation value of a generic observable $A$ generalizing the Bell stationary one definition

$$
\overline{A(\boldsymbol{a}, \lambda)}=\int A(\boldsymbol{a}, \lambda) \rho(\lambda) \mathrm{d} \lambda
$$

in the following way

$$
\overline{A(\boldsymbol{a}, \lambda+i \Delta \lambda(t))}=\int_{\gamma(t)} A\left(\boldsymbol{a}, \lambda+i \Delta \lambda\left(t^{\prime}\right)\right) \rho\left(\lambda+i \Delta \lambda\left(t^{\prime}\right)\right) \mathrm{d}\left(\lambda+i \Delta \lambda\left(t^{\prime}\right)\right) .
$$

where $\boldsymbol{a}$ is the versor parallel to the magnetic field of the detector, $A$ is the observable to be measured and the integral is a path dependent one in the complex plane (or complex hyper-planes) of the generalized hidden variable complex space $\lambda$ averaged on the time dependent density distribution $\rho$.

We assume, for example, that the hidden variable describing the spin is a complex time dependent vector making spin projected angles experimental measured not perfectly defined but acquiring an imaginary non-stationary component (dependent on atomic energy variable bandwidth or on nucleus time dependent mass defect).

Consequently, we have to take into account a coherent time dependent generalization of Bell two-particle correlation function of local hidden variable models, assuming that the probability density distribution is not stationary.

$$
P(\boldsymbol{a}, \boldsymbol{b})=\int A(\boldsymbol{a}, \lambda) B(\boldsymbol{b}, \lambda) \rho(\lambda, t) \mathrm{d} \lambda
$$

This classical time dependent correlation function must be compared with that one stationary predicted by Quantum Mechanics

$$
P_{Q, M .}(\boldsymbol{a}, \boldsymbol{b})=-\boldsymbol{a} \cdot \boldsymbol{b}=-\cos (\boldsymbol{a}, \boldsymbol{b})
$$

where $\boldsymbol{a}$ e $\boldsymbol{b}$ are the standard versors of the axis of the magnetic fields of each detector. We interprete the introduced non-stationary density distribution $\rho$ as a time dependent, or memory dependent, sub-ensemble partition of the distinguishable elements of the ensemble of detected pairs of entangled particles (this assumption implies that detectors count rates may have hidden memory ef- 
fects).Since we don't assume that the entanglement of two unstable particles satisfy perfect anti-correlation (the apparatus and the dynamic vacuum break in our approach the rotational symmetry), the correlation defined in (4) follows the generalized relation dependent on an unknown function $f$ (whose determination will be studied in a work the author is preparing)

$$
P(\boldsymbol{a}, \boldsymbol{a})=-f(\Delta \lambda(t))
$$

Instead of the standard one

$$
P(\boldsymbol{a}, \boldsymbol{a})=-1
$$

which is used in recent similar papers on Bell's Theorem and supporting Einstein local realism [11] [12] [13].

We remark that it may be possible to test directly our proposal conceiving experiments with oscillating axis of magnetizations to be described by complex versors $\boldsymbol{a}(t)$ and $\boldsymbol{b}(t)$, to be inserted in Equation (5), which make Quantum Mechanics predicted correlation functions asymptotically cosinusoidal.

Our framework could recover the standard stationary Bell correlation functions if the versors dynamics is ergodic; in fact the E.P.R. experimental tests are just a by-product of a finite discrete time series of a great number of data sets, which converges asymptotically to the quantum mechanical ensemble statistical averages, if it is valid for these numerical sets the law of great numbers, that is by assuming

$$
P(a, b)=\lim _{N \rightarrow \infty} \frac{1}{N} \sum_{n=1}^{N} a_{n} b_{n} .
$$

The Copenhagen interpretation of Quantum Mechanics works as long as $P$ of (8) converges to the standard relation (5).

To make our hidden variable model experimentally testable we have to introduce a similar time dependence in the C.H.S.H. hidden variable correlation function $[17]$

$$
E_{\text {Bell }}(\boldsymbol{a}, \boldsymbol{b})=P_{++}(\boldsymbol{a}, \boldsymbol{b})+P_{--}(\boldsymbol{a}, \boldsymbol{b})-P_{+-}(\boldsymbol{a}, \boldsymbol{b})-P_{-+}(\boldsymbol{a}, \boldsymbol{b})
$$

which for Hilbert quantum states satisfies

$$
E_{Q . M}=\cos 2 \alpha
$$

(this relation cannot be deduced for the unstable states described by Gamow ket states), where $\alpha$ is the relative angle between the magnetic fields of the two detectors.

Our model can be falsified by testing the following generalized relation

$$
E(\boldsymbol{a}, \boldsymbol{b})=E_{\text {Bell }}(\boldsymbol{a}, \boldsymbol{b}) F(\Delta \lambda(t)) .
$$

where the unknown function $F$ must be determined by a future self consistent model. This relation can be compared with the Quantum Mechanics predicted violation by imposing the following asymptotic limit

$$
\lim _{t \rightarrow+\infty} F(\Delta \lambda(t))=\cos 2 \alpha
$$


where $\alpha$ is the relative angle between the two detector axis.

Therefore our alternative approach based on local complex hidden variable doesn't satisfy anymore at each instant $t$ the C.H.S.H. inequality [17]

$$
S \leq 2
$$

where the parameter $S$ is defined as usual by

$$
S=\left|E(\boldsymbol{a}, \boldsymbol{b})-E\left(\boldsymbol{a}, \boldsymbol{b}^{\prime}\right)\right|+\left|E\left(\boldsymbol{a}^{\prime}, \boldsymbol{b}\right)+E\left(\boldsymbol{a}^{\prime}, \boldsymbol{b}^{\prime}\right)\right| .
$$

We think that by inserting the generalized $E$ defined in (11) in the second member of (14), the relation (13) is violated, showing that our model is compatible with the experimental tests supporting violations of Bell Inequalities.

We outline that the standard formalism assumes stationary expectation values since it is assumed the postulate of instantaneous collapse of the wavefunction; on the contrary, if one considers finite time spin measurements it follows that the detector clicks can be influenced from what happened in the past. The need to extend local hidden variable models to the measurement process expressed by Bell and his followers was implemented testing in the laboratory the C.H.S.H. inequality with the pragmatic relation [16]

$$
S \leq \frac{4}{\eta}-2
$$

where $\eta$ is the detector efficiency parameter that is always less than one for real detectors (making it equivalent to the standard inequality violated in E.P.R. experiments). We note that our generalized time dependent C.H.S.H. function $S$ can be recasted as a detection efficiency dependent function of (13) imposing the following condition

$$
F(\Delta \lambda(t))=\frac{4}{\eta(\Delta \lambda(t))}-2=S\left(E_{Q . M .}(\alpha+i \Delta \alpha(t))\right)
$$

where the imaginary relative angle inserted in the function $E$ of formula (10) takes into account that the spin of unstable particles is not a well defined real value since it depends on the hidden fluctuating energy bandwidth. Although our proposal is not a self consistent model, since it depends on the unknown function $f$ and $F$, introduced in (6) and (11), we hope will stimulate other authors to look for a realistic description of the spin of accelerated unstable systems, that has till now based on the Pauli operatorial formalism describing the spin of free isolated electrons (whose extension in accelerated frame and for extended particles is still lacking).

We note over more that relation (13) is usually tested in anon inertial frame that is the rotating Earth, where is present the background gravity field too. Therefore, we think, it should be generalized the formula of the detector efficiency $\eta$ making it dependent on the Earth gravity and rotation and on the vacuum index induced by background fields

$$
\eta=\eta(\boldsymbol{g}, \boldsymbol{a}, n(x, t))
$$

where $n(x, t)$ is the vacuum index of hidden fast induced vacuum polariza- 
tion; we interpret it as caused by background fields of accelerated and radiating unstable quantum systems. We wish we will be able to develop more deeply in a future work an infinite dimensional hidden variable model of non relativistic vacuum fluctuations.

We believe that path integral averages on the infinite dimensional space of vacuum indexes might be useful for a deterministic reformulation of finite time photon pair creation and annihilation and maybe, more generally, of Q.E.D. theory in accelerated frames (that has been so far successfully tested not in an inertial frame but in the Earth rotating frame of reference).

We think that this new theoretical framework could be experimentally tested, comparing its predictions with the standard one, once it is developed a selfconsistent wave pilot wave model of vacuum fluctuations with memory. This alternative model could describe exactly the time dependence of the imaginary hidden variables, implementing, we suggest, a continuous space time description of the spin variable caused by hidden path memory dependent forces [10].

We believe that this research program might allow to make more accurate experimental tests of the validity in nuclear physics of the uncertainty principle and of the instantaneous wave function collapse postulate looking for path memory dependent nucleon impulses and forces. We want to remind once more that till now every Bell tests have been conceived for presumed isolated stable systems, disregarding gravity induced vacuum entanglement [18] and the dynamic decoherence effect due to time dependent background fields. On the contrary, we believe, as suggested recently by some authors [19] [20], that it is necessary to look for hidden variable models of vacuum fluctuations (and its coupling with the apparatus) and to speculate on infinite dimensional generalizations of Bell's Theorem for unstable particles viewed as excitation states of unstable vacuum, exploiting the new concept of dynamic vacuum [21]. Therefore we hope that our tentative proposal will stimulate researchers to go beyond the abstract formalism of Quantum Mechanics used so far for describing the spin of extended accelerated nucleons and nuclei as quantized eigenvalues of non-commutating operators and will be useful for describing more generally the entanglement of quantum systems in non inertial frames [22].

\section{Conclusion}

We illustrate in this work our tentative proposal aimed to implement the realistic approach of Einstein to Quantum Mechanics in the context of nuclear physics, where the standard probabilistic description concerns the dynamics of decaying unstable quasibound nucleons. We propose to generalize Bell local hidden variable models to such general unstable quantum states introducing complex time dependent hidden variables vectors and angles and time dependent expectation values, which could give a classical explanation of Bell inequalities violations, by the introduction of complex time dependent spin vectors. We suggest to compare experimentally the asymptotic temporal limits of this path 
memory dependent averages with the standard stationary predictions deduced from Bell's Theorem, conceiving experiments of Stern-Gerlach apparatus with oscillating magnetic axis. Finally, we hope that this new tentative approach to hidden variable models could be applied to develop a deterministic model of every microscopic open quantum system (such as decaying nuclei or excited interacting atoms) and could, more generally, shed new light on the incompleteness of the standard Quantum Mechanics formalism for describing the dynamics of extended accelerated particles in a dynamic vacuum.

\section{Conflicts of Interest}

The author declares no conflicts of interest regarding the publication of this paper.

\section{References}

[1] Einstein, A., Podolsky, B. and Rosen, N. (1935) Can Quantum Mechanical Description of Physical Reality Be Considered Complete? Physical Review, 47, 770-780. https://doi.org/10.1103/PhysRev.47.777

[2] Schrödinger, E. (1935) Die gegenwärtige Situation in der Quantenmechanik. Naturwissenschaften, 23, 807-812. https://doi.org/10.1007/BF01491891

[3] Von Neumann, J. (1932) Mathematical Foundations of Quanatum Mechanics. Princeton University Press, Princeton.

[4] Bohm, D. (1952) A Suggested Interpretation of the Quantum in Terms of Hidden Variables I. Physical Review, 85, 166-179. https://doi.org/10.1103/PhysRev.85.166

[5] Bell, J.S. (1964) On the Einstein Podolsky Rosen Paradox. Physics Physique Fizika, 1, 195-200. https://doi.org/10.1103/PhysicsPhysiqueFizika.1.195

[6] Bohr, N. and Wheeler, J.A. (1939) The Mechanism of Nuclear Fission. Physical Review, 56, 426-450. https://doi.org/10.1103/PhysRev.56.426

[7] Gamow, G. (1928) Zur Quantentheorie des Atomkernes. Zeitschrift für Physik, 51, 204-212. https://doi.org/10.1007/BF01343196

[8] Bei, G. (2021) A Hidden Variable Model of Unstable Quantum States. Physical Science \& Biophysics Journal, 5, Article ID: 000171.

https://doi.org/10.23880/psbj-16000171

[9] Bei, G. (2021) A Hidden Variable Model of Nuclear Reactions. International Journal of Applied Physics, 8, 1-4. https://doi.org/10.14445/23500301/IJAP-V8I2P101

[10] Bei, G. (2021) A Deterministic Model of Path Memory Dependent Inter-Nucleon Forces. Physical Science \& Biophysics Journal, 5, Article ID: 000178. https://doi.org/10.23880/psbj-16000178

[11] Sica, L. (2020) The Bell Inequalities: Identifying What Is Testable and What Is Not. Journal of Modern Physics, 11, 725-740. https://doi.org/10.4236/jmp.2020.115047

[12] Chen, G.Y. (2019) Collapse of Bell's Theorem. Journal of Modern Physics, 10, 1157-1165. https://doi.org/10.4236/jmp.2019.1010076

[13] Hess, K., De Raedt, H. and Michielsen, K. (2017) Analysis of Wigner's Set Theoretical Proof for Bell-Type Inequalities. Journal of Modern Physics, 8, 57-67. https://doi.org/10.4236/jmp.2017.81005

[14] Jung, K. (2020) Polarization Correlation of Entangled Photons Derived without Using Non-Local Interactions. Frontiers in Physics, 8, Article No. 170. 
https://doi.org/10.3389/fphy.2020.00170

[15] Khrennikov, A. (2021) Quantum Postulate vs. Quantum Nonlocality: On the Role of the Planck Constant in Bell's Argument. Foundations of Physics, 51, Article No. 16. https://doi.org/10.1007/s10701-021-00430-3

[16] Nordén, B. (2016) Quantum Entanglement: Facts and Fiction-How Wrong Was Einstein After All? Quarterly Reviews of Biophysics, 49, e17. https://doi.org/10.1017/S0033583516000111

[17] Clauser, J.F., Horne, M.A., Shimony, A. and Holt, R.A. (1969) Proposed Experiment to Test Local Hidden Variable Theories. Physical Review Letters, 23, 880-884. https://doi.org/10.1103/PhysRevLett.23.880

[18] Xu, Q., Ahmad, S.A. and Smith, A.R. (2020) Gravitational Waves Affect Vacuum Entanglement. Physical Review D, 102, Article ID: 065019. https://doi.org/10.1103/PhysRevD.102.065019

[19] 't Hooft, G. (2021) Fast Vacuum Fluctuations and the Emergence of Quantum Mechanics. Foundations of Physics, 51, Article No. 63.

https://doi.org/10.1007/s10701-021-00464-7

[20] Suzuki, Y. and Mertes, K.M. (2019) Vacuum Texture: A New Interpretation of Quantum Mechanics and a New Loophole for Bell's Inequality Measurements that preserves Local Realism and Casuality.

[21] White, H., Bailey, P., Lowrence, J., George, J. and Vera, J. (2019) A Discussion on a Dynamic Vacuum Model: Derivation of Helmoltz Equation from Schrödinger Equation. Physics Open, 1, Article ID: 100009.

https://doi.org/10.1016/j.physo.2019.100009

[22] Toroš, M., Restuccia, S., Gibson, G.S., Cromb, M., Ulbricht, H., Padgett, M. and Faccio, D. (2020) Revealing and Concealing Entanglement with Non-Inertial Motion. Physical Review A, 101, Article ID: 043837.

https://doi.org/10.1103/PhysRevA.101.043837 